\title{
Primary care provider approaches to preventive health delivery: a qualitative study
}

\author{
Hemalatha Murugan ${ }^{1}$, Clarence Spigner ${ }^{1}$, Christy M. McKinney ${ }^{2}$ and Christopher J. Wong ${ }^{3}$ \\ ${ }^{1}$ School of Public Health, University of Washington, Seattle, WA, USA \\ ${ }^{2}$ Department of Pediatrics, School of Medicine, Division of Craniofacial Medicine, University of Washington, \\ Seattle, WA, USA \\ ${ }^{3}$ Department of Medicine, Division of General Internal Medicine, University of Washington, Seattle, WA, USA
}

\begin{abstract}
Aim: The objective of this study was to seek decision-making insights on the provider level to gain understanding of the values that shape how providers deliver preventive health in the primary care setting. Background: The primary care clinic is a core site for preventive health delivery. While many studies have identified barriers to preventive health, less is known regarding how primary care providers (PCPs) make preventive health decisions such as what services to provide, under what circumstances, and why they might choose one over another. Methods: Qualitative methods were chosen to deeply explore these issues. We conducted semi-structured, one-on-one interviews with 21 PCPs at clinics affiliated with an academic medical center. Interviews with providers were recorded and transcribed. We conducted a qualitative analysis to identify themes and develop a theoretical framework using Grounded Theory methods. Findings: The following themes were revealed: longitudinal care with an established PCP-patient relationship is perceived as integral to preventive health; conflict and doubt accompany non-preventive visits; PCPs defer preventive health for pragmatic reasons; when preventive health is addressed, providers use multiple contextual factors to decide which interventions are discussed; and PCPs desired teambased preventive health delivery, but wish to maintain their role when shared decisionmaking is required. We present a conceptual framework called Pragmatic Deferral.
\end{abstract}

Key words: decision-making; prevention; preventive care; primary care; qualitative research

Received 19 June 2017; revised 20 November 2017; accepted 26 November 2017;

first published online 8 January 2018

\section{Background}

Achieving better outcomes for a population through preventive measures remains a major healthcare goal (Ockene et al., 2007). Despite preventive health successes such as the reduction in cardiovascular outcomes (Mozaffarian et al., 2016) and the decline in vaccine-preventable infectious diseases (Roush et al., 2007), opportunities for improvement remain. Among the top five causes of mortality in the United States, an

Correspondence to: Christopher J. Wong, Associate Professor, Department of Medicine, Division of General Internal Medicine, University of Washington, 4245 Roosevelt Way NE, Box 354760, Seattle, WA 98105, USA. Email: cjwong@uw.edu estimated $20-40 \%$ of deaths are potentially preventable (Yoon et al., 2014). While preventive health encompasses a broad range of activities, settings, and providers - including employer-based programs, direct appeals through mass marketing (Elder, 2014), public health campaigns, and legislation such as tobacco taxes (Chaloupka et al., 2012) - much of this important work continues to take place at the clinician's office. The optimal means to deliver preventive health within the primary care setting continues to be debated. Key questions include what services should be offered, in what type of office visit, and by whom.

The number of recommended preventive services issued is daunting. For example, the United States Preventive Services Task Force (USPSTF), 
which issues guidelines regarding preventive health, recommends 52 services at the grade ' $\mathrm{A}$ ' or ' $\mathrm{B}$ ' levels (moderate to high certainty of benefit) (U.S. Preventive Services Task Force, 2017). Other organizations such as the Advisory Committee on Immunization Practices, the Institute of Medicine, and subspecialty societies issue their own guidelines. The primary care provider (PCP) must review these recommendations and decide what preventive services to offer.

Even if a set of preventive services is agreed upon, the optimal time to offer them is uncertain. Office visits may generally be divided into two categories: separate, stand-alone preventive health appointments ('preventive health visits' or 'wellness visits') or visits for medical conditions. Preventive health may be delivered during either type of visit. As delivering preventive services takes considerable time (Medder et al., 1992; Yarnall et al., 2003) and real-world delivery is inefficient (Kottke et al., 1993; Ruffin et al., 2000; Stange et al., 2000; Cosgrove, 2012), the dedicated preventive visit is one method for PCPs to ensure adequate attention to preventive health. The overall rate of preventive visits in the United States in 2012 was 61 per 100 persons, with variability by age, gender, and state (Hing and Albert, 2016). PCPs report being more likely to provide patients preventive medicine during wellness visits than during acute visits (Snipelisky et al., 2016), and increased screening rates have been associated with health maintenance visits (Ruffin et al., 2000). The Center for Medicare and Medicaid Services accordingly has created the Annual Wellness Visit for Medicare beneficiaries. Yet there continues to be conflicting evidence and differences of opinion regarding the efficacy of preventive health visits, with critics citing insufficient evidence for mortality outcomes and proponents arguing for other benefits (Boulware et al., 2007; Krogsboll et al., 2012; Society of General Internal Medicine, 2013; Wong et al., 2014).

In addition to these decisions of what preventive services to offer and when to offer them, there are evolving concepts as to which personnel should be involved in performing preventive healthcare. Enlisting other members of the healthcare team has been proposed as a means to improve preventive health delivery. 'Share the Care' (Gupta et al., 2016) and Patient Centered Medical Home (PCMH) models (U.S. Department of Health and
Human Services, 2016) use non-PCP staff to ensure that a patient's preventive health recommendations are met. Despite ample literature addressing challenges to PCMH implementation (Rodriguez et al., 2014) and role assignment within teams (Edwards et al., 2015), there has been relatively less attention paid to exploring how PCPs wish their roles to be within healthcare teams when providing preventive healthcare.

This study sought to add to the current understanding of preventive health in the office-based setting by learning more about the perspective of PCPs in making decisions about what preventive health to offer, why they might choose to address preventive health during one visit but not another, and how they see their role in healthcare teams. Although previous qualitative studies have addressed perceived barriers and facilitators to specific preventive health topics (Simmons et al., 2016; Abraham et al., 2017), few have directly asked PCPs how they act as agents of preventive health delivery. Given the open-ended nature of our questions, we determined that a qualitative approach was optimal.

\section{Methods}

\section{Research design}

We aimed to explore the dynamics of preventive care delivery by PCPs using an approach procedurally informed by the principles of Grounded Theory (Creswell, 2007; Corbin and Strauss, 2015) with no theoretical assumptions. Earp and Ennett (1991) point to how 'conceptual diagrams are used to organize and synthesize knowledge, defined concepts, provide explanation for causal and associative linkages, generated hypotheses and specific research questions, plan and target interventions, designated variables to be operationalized, and anticipate analytical approaches.' Our purpose was therefore to develop a conceptual model to help explain the decision-making process of preventive care delivery by primary care health providers.

\section{Sample and recruitment}

We defined PCPs as physicians, advanced registered nurse practitioners (ARNPs), or physician assistants who had a panel of patients for whom they were the responsible longitudinal care provider. We recruited PCPs from primary care 
clinics affiliated with the University of Washington, an academic medical center in Seattle, Washington, USA. These clinics were group practices in an urban setting. Subjects could work at any amount of clinical full-time equivalent (FTE). Upon receiving study approval from the Institutional Review Board, we approached primary care clinic directors to obtain permission to introduce the study at administrative meetings at those clinics and to invite PCPs to take part in the study. With the clinic director's permission, we followed up with no more than three recruitment emails to PCPs. In total, 21 subjects from four academic clinics ultimately agreed to participate in our study.

\section{Data collection}

At the beginning of each face-to-face interview, we provided the subject with a brief overview of the study purpose, asked permission to audiorecord the session, and obtained written consent. Basic demographics were gathered at the start of each session. The interview guide consisted of three topic areas related to prevention services performed during different types of visits and team-based healthcare (Table 1). The questions in the interview guide were developed based on literature review and designed to elicit answers to the research questions. Interviewers asked followup questions as needed for clarification and to probe for meaning. Additional questions pertained to the electronic medical record (EMR) and were not included as part of this analysis. All interviews were conducted by two of the authors between July 2015 and September 2015. Each interview session lasted $\sim 20 \mathrm{~min}$.

\section{Data analysis}

All interviews were audio-recorded and transcribed verbatim for content analysis of the transcripts (Creswell, 2007). Our analysis used the following iterative steps: (1) Three members of the research team independently read and re-read each transcript in order to achieve as broad an understanding of the content as possible. (2) Each member of the team independently conducted open coding and organized the data into subcategories. Given the sample size, all coding was done by hand. (3) All team members met face-to-face to discuss their reasons for assigning their codes, and to agree on a list of subcategories.
Table 1 Summary of key protocol questions for interviews

Topic A

(1) When you address preventive health with your patients, what guidelines do you typically follow, and why?

(2) There are many different preventive services recommended by various organizations. How do you prioritize what preventive health to cover during a preventive health visit?

(3) During preventive visits, do you typically address a patient's other conditions, if they have other health conditions?

Topic B

(1) In a non-preventive visit, would you typically check whether any of the patient's preventive health is upto-date?

(2) Keeping in mind that there are many different types of preventive services, from cancer screening to immunizations, etc., are there services that you prioritize certain services over others during these non-preventive visits? (and if so, why?)

(3) If you do not typically offer preventive health services during a non-preventive visit, why not?

(4) If you are seeing a patient in follow up of diabetes and hypertension rather than an acute visit for shoulder pain, how would this affect whether you address preventive health?

(5) For either scenario, what if the patient is not your primary care patient - does this affect whether you would be more likely to check a patient's preventive health during a non-preventive health visit?

Topic C

(1) Are there preventive services you feel would be appropriately addressed by non-physician and nonARNP staff? If so, which ones, and why?

(2) Are there preventive health services that you would uncomfortable with an MA-level training staff member addressing with a patient? If so, which ones, and why?

(4) In an iterative process, a matrix was created to sort the codes into subcategories which were then grouped into clusters, out of which themes emerged.

\section{Validation and saturation}

Inter-rater reliability (Creswell, 2007) was reinforced by having the two physician members of the team independently code each transcript and a third qualitative research expert and nonphysician code these same transcripts within a similar time for triangulation. Where there was disagreement between coders, discussion ensued until either consensus was reached or the code was discarded. Saturation was reached when no new information was forthcoming from the data. 


\section{RESULTS}

\section{Demographics}

The 21 in-depth interviews included 17 physicians and four ARNPs. Subjects practiced at four different clinics affiliated with an academic medical center. These clinics included a women's healthcare clinic, a university general internal medicine clinic, and teaching clinics at a county hospital. The average age of the primary care health providers was 48 years. In total, $71 \%$ of the participants were women and the average clinical FTE was half-time (Table 2).

Theme: longitudinal care with an established PCP-patient relationship is perceived as integral to preventive care

All clinic sites in this study were group practices. Thus providers routinely saw not only their own patients but also patients assigned to other providers. When seeing another provider's primary care patient in a clinic visit, subjects in this study strongly preferred that most preventive services be discussed between the patient and his or her own $\mathrm{PCP}$, as reflected in these typical responses:

'And for complicated preventive health ... if the patient is not my primary care patient, then I usually don't do [it]. I ask them to come back and talk with their doctor rather than I do it. Particularly for cancer screening, you need to evaluate the patient and assess whether he needs cancer screening or not.'

(Interviewee 15)

'It's a waste of my time to check, if a patient is not my primary patient.'

(Interviewee 6)

Subjects considered that involvement of the patient's own PCP was essential to preventive health because of the need to understand the patient's unique values and beliefs. They regarded

Table 2 Characteristics of subjects

\begin{tabular}{ll}
\hline Number of subjects & 21 \\
Age (mean, years) & 48 \\
Women (\%) & 71 \\
Years in practice (mean) & 17 \\
Clinical full-time equivalent (mean) & 0.51 \\
\hline
\end{tabular}

that the PCP was in the best position to have this knowledge:

'I typically don't address preventive service if it is not my primary patient... I think it is harder to know [the] patient's knowledge, philosophy and beliefs in that shorter conversation. I think it is harder to do that if you don't have that relationship.'

(Interviewee 11)

'If it's another provider's patient and you know they're due for a colon cancer screening or it looks like they've declined multiple preventive things in the past ... it seems like a conversation that they really need to have with their primary doctor...'

(Interviewee 5)

'There's something about the doctor-patient relationship that is better for somebody to have one doctor who knows them...'

(Interviewee 1)

This utmost respect for the longitudinal relationship drove clinicians to defer preventive health to another visit if that relationship was not present.

Theme: conflict and doubt accompany non-preventive visits

In contrast to a dedicated preventive health visit, during acute or chronic care visits providers must consider whether to address preventive health at all. We found that providers expressed a desire to accomplish some preventive health during non-preventive visits, but also felt discomfort at either falling short due to time constraints or being only able to focus on brief interventions. The following responses represent this theme:

'But I'm not, I'll be honest, I'm not consistent in what I do for preventive health in my nonpreventive visits.'

(Interviewee 1)

'...it's one of those things I think I should do.'

(Interviewee 4)

In other words, providers felt that they should address preventive health in different types of 
non-preventive visits, but often felt that they did not or could not do so effectively.

Theme: PCPs defer preventive health for pragmatic reasons

We found that despite this conflict, PCPs nevertheless forged ahead and acted pragmatically to address more pressing concerns and often deferred preventive health to another time:

'If you're talking about 3 or 4 acute problems, there might be no time for anything else.'

(Interviewee 17)

'If the patient has [an] acute problem basically I deal with that first and if I have enough time I will address preventive health during that visit.'

(Interviewee 15)

'It is all based on time. I will have the health maintenance tab open. I do prep the night before and I write the things down to address. But if the patient comes in late, then I will skip it.'

(Interviewee 6)

'With a patient ... who has so many different problems, usually the preventive health is low priority. Or the last priority if there's time at the end. I don't attempt to really do more than one or two preventative interventions, and often just save it and chip away at it each visit if I can.'

(Interviewee 17)

While deference was the norm, for measures that were perceived as either low risk or relatively quick, such as immunizations, they would make exceptions:

'So, if it's flu season and a patient always gets a flu shot and says "Hey could I get my flu shot while I'm here?"-sure.'

(Interviewee 9)

'The tests that are pretty easy to get, I don't have to spend much time on, I will say " $\mathrm{Hi}$, we're going to check this, this, and this and I put those orders in.'

(Interviewee 19)

Providers who routinely conducted separate preventive visits valued the time these visits afforded to focus on prevention.
Theme: when preventive health is addressed, providers use multiple contextual factors to decide which interventions are discussed

Once the decision is made to address prevention, regardless of visit type, then providers must choose what services to address. While some providers simply reported providing a patient an entire list of what preventive health measures are 'due', in most cases PCPs attempted prioritization. Providers cited a number of factors, including patient preferences, the patient's health conditions, prompts from the EMR, local quality improvement initiatives, and the perceived impact of a preventive service:

'...I try to tailor the visit to be what the patient sort of wants and expects.'

'I use age- and gender-based recommendations and then depending on the patient like how healthy they are or they are not.'

(Interviewee 18)

'I individualize the guidelines depending on the overall health of the patient. If someone is extremely ill from serious multiple diseases and if the life expectancy is short then of course I will not be doing cancer screening.'

(Interviewee 16)

'I'm also influenced some by what the quality measures are that the clinic is being graded on.'

(Interviewee 18)

For guidance, most of the providers in these clinics used the USPSTF, citing that 'compared to other societies they are less biased' and 'they are the most evidence-based'. But, they also used multiple guidelines, often for topics not addressed by the USPSTF or if there were competing guidelines they felt the patient should be aware of.

Among preventive services, these providers in general prioritized cancer screening. Reasons for this included their perception of patient concerns:

'I will say that I always, I pretty much always address cancer screening...the fear of cancer's so huge in our society, and clearly I would not want to miss a cancer...'

(Interviewee 8) 
In contrast, other screening services might be considered valuable but lower priority: '...missing things like a hearing problem - probably not the end of the world'. In addition, screening for depression was generally conducted less frequently because it was felt to be symptomatic if present:

'If I know the patient, if they are my patients, if I know them for years, I probably don't do any psychosocial screening for depression or anxiety during a preventive health visits because I know that person.'

(Interviewee 16)

'I get into psychological screening and other issues secondarily. Usually... honestly when I think of it. I mean, but I would usually be triggered by other factors...'

(Interviewee 14)

Thus there was no single guiding principle for selecting preventive health services, but rather a mix of multiple considerations.

Theme: PCPs desired team-based preventive health delivery, but wish to maintain their role when shared decision-making is required

Nearly all providers expressed an interest that other members of the healthcare team such as nurses or medical assistants be involved in their patients' preventive healthcare. They did not wish however to cede all preventive healthcare to other team members. The more controversial or complex the preventive health topic, the more the providers wanted to maintain primacy in discussion with the patient. For example, many providers supported a role for a medical assistant to independently address immunizations, but for cancer screening, they showed discomfort if the PCP was not involved:

'For mammogram and colonoscopy, things are too nuanced for someone not specifically trained in it, so I would say someone being specifically trained is good.'

(Interviewee 10)

'I think the mammogram discussion ... it's very nuanced and... the guidelines differ so much...I think I would want to have the initial discussion.'

(Interviewee 1)
'I think for cancer screening it would be appropriate if it is addressed by a physician. For vaccines, it is okay if they [the medical assistants] are letting you know if it is pending.'

(Interviewee 19)

These providers were comfortable with a staff member checking and suggesting preventive health measures that required shared decisionmaking, as long as the PCP still worked directly with the patient to establish the appropriate screening measure and interval.

\section{Discussion}

This qualitative study adds to the existing literature by not only describing the barriers that PCPs face, but also presenting a model of PCPs as active decision-makers in their practice. We describe how they make decisions as to when to offer preventive services, which ones to address, and why, and how they view their role as part of a care team.

When addressing prevention, we uncovered a steadfast adherence to the longitudinal patient PCP relationship regardless of other barriers or constraints. This modest but important distinction emerged in several contextual situations, such as when there were concerns about having limited knowledge of another PCP's patient, and when there were concerns about conducting adequate shared decision-making. These clinics all had EMRs - the value of the longitudinal care relationship is therefore in information not always accessible in the chart, concordant with prior findings that interpersonal communication and coordination of care scale scores were associated with being more up-to-date on screening services and health habit counseling (Flocke et al., 1998).

Do providers place value in addressing prevention during illness visits? The issue of visit type preventive visits versus those for acute or chronic illnesses - remains important. It may be easy to forget how much healthcare has changed - prior studies labeled the addressing of prevention during chronic care or illness visits as the 'opportunistic method', and in a previous era many physicians did not endorse this approach at all (Rebelsky et al., 1996). In contrast, subjects in this study clearly valued addressing prevention, and felt that they should address it routinely. 
The theme of conflict and doubt arose accordingly in settings in which they felt they could not do so adequately.

Exploring the reasons for such doubt led to the theme of pragmatism. Several studies have examined delivery of preventive services during nonpreventive visits with regard to how infrequently it occurs (Kottke et al., 1993; Stange et al., 1998; 2000), what observed factors are associated with addressing prevention (Flocke et al., 1998), and what communication methods are used (Cohen et al., 2004). Most studies however did not address why providers choose to address preventive health in a given clinic visit, and what factors they perceive as being relevant. Our study explores this concept further by asking providers directly how they make decisions regarding when to address prevention in non-preventive visits. We found that PCPs consistently wrestled with prioritizing nonpreventive versus preventative care during nonpreventive visits. Similar to previous studies, time constraints continue to be a major factor in deciding whether to address preventive health (Burack, 1989; Kottke et al., 1993; Cornuz et al., 2000; Yarnall et al., 2003). While our subjects expressed feelings of exasperation and vexation in their desire to deliver preventive care, these providers were not overwhelmed by these constraints. Rather, their decision-making was characterized by an underlying pragmatism, grounded in the circumstances of the patient. These PCPs evaluate each clinic visit and prioritized and triaged care based on the particular or unique circumstances of the individual patient. Barriers to preventive care are realized in that the immediate circumstances of the patient may have to take priority, consequently hindering or giving less priority to preventive care. Such real-world conditions meant that these providers practiced a constant state of triage with regard to fitting in preventive health.

The decision to choose one preventive service over another is complex. For example, one could postulate that providers use 'cost-effectiveness', 'institutional priorities', or 'patient-centeredness' as a rubric to prioritize which screening tests or other preventive interventions to offer. An interesting finding in our study was that there was in fact no overriding priority system. Instead, providers typically started with a foundation based on recommendations of the U.S. Preventive Services Task Force, and then prioritized based on the available time, the patient's overall health, a preventive service's perceived complexity, the patient's agenda, and the general sense that cancer screening is expected on the part of patients.

Finally, who is the appropriate healthcare provider to deliver preventive health? This study revealed that PCPs continue to have a profound determination to maintain their role in providing preventive care to their patients. The use of team members engaging with the patient is an attractive approach to increase preventive service delivery (Pelak et al., 2015). PCPs readily acknowledged the effectiveness of other staff members such as medical assistants to help increase the flow of patients through the healthcare delivery process. Rose et al. (2015) point to the effectiveness of prescreening for patients who are registered for nonacute visit in an academic medical clinic similar to the sites in our study. However, as the 'team' approach proliferates, our study shows that PCPs strongly value that a patient's PCP should be involved in complex preventive care decisions, especially for topics such as cancer screening, in which appropriate responses to patients' questions would demand the time and expertise of the PCP.

\section{Conceptual model}

We undertook this exploratory study to unearth a conceptual framework or model of decisionmarking around delivering preventive health recommendations in a clinic-based setting (Earp and Ennett, 1991). Squires et al. (2016) employed a similar qualitative strategy and built their conceptual model for public health economic evaluation. Their study was informed by literature reviews of key challenges in public health economics. In our study, a framework emerged to illustrate how PCPs acted due to the realities the patients' needs. This model can be described as 'Pragmatic Deferral'. As shown in Figure 1, in a non-preventive visit, multiple factors tend to tilt the momentum of a visit away from including preventive care. While time continues to be a factor, time constraints emerged as part of a larger model of decision-making. These include the presence of uncontrolled health conditions, prevention not being part of the visit agenda, not being the patient's primary longitudinal care provider, or simply not having time. If there is time, then the services delivered are more likely to be short and 


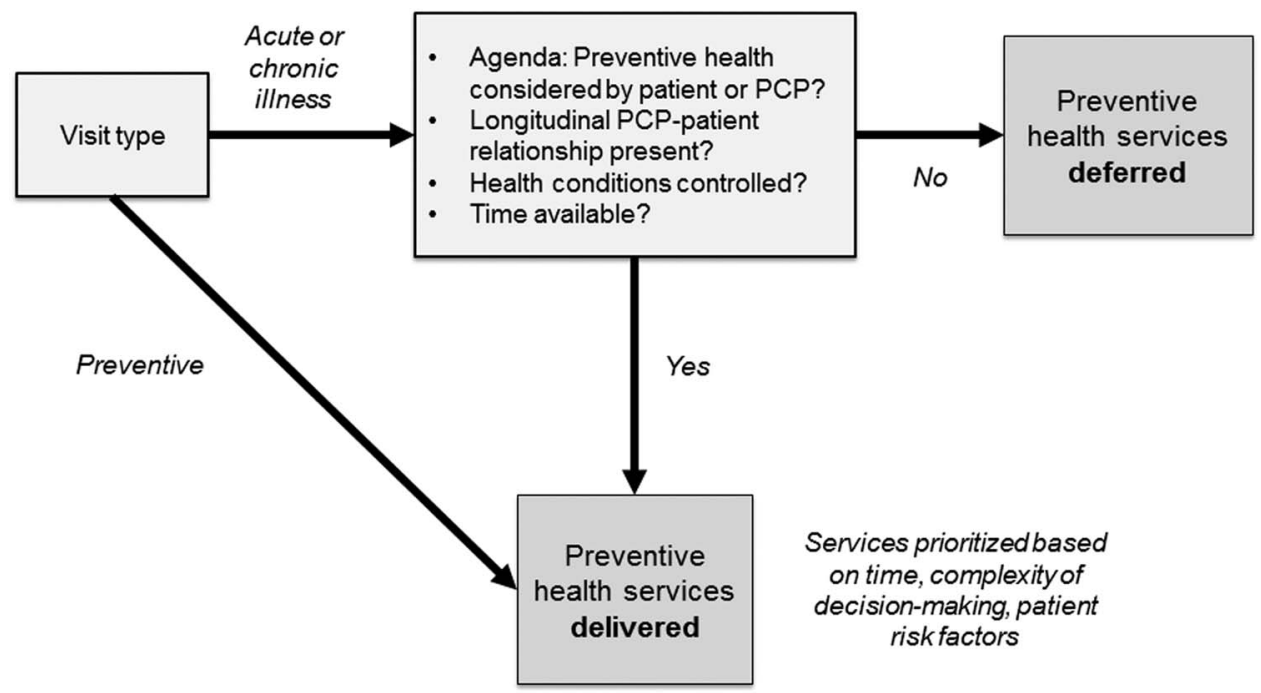

Figure 1 Conceptual framework: 'Pragmatic Deferral'. PCP $=$ primary care providers

require less shared decision-making (eg, vaccinations instead of a cancer screening discussion). The barriers and facilitators described in this and prior studies modulate these factors.

This model echoes and builds upon prior work. A pilot study of prostate cancer screening found similar complex barriers and facilitators (Guerra et al., 2007), and one theory pertaining to decisionmaking in cancer screening describes the process as 'Making the Most of the Visit' (Starks and Trinidad, 2007). Our model addresses prevention as a whole, rather than focusing on a single topic such as cancer screening. Mirand et al. (2003) similarly found that prioritizing 'secondary' care issues was a barrier to addressing prevention, and also uncovered the theme of providers' selfperceived role as a 'one-stop-shop' or 'savior' as a barrier, a concept that did not arise in our analysis. Jaen et al. (1994) over 20 years ago developed a theoretical 'Competing Demands' model in which the factors influencing preventive health delivery are the patient, physician, and clinical setting. Our model identified similar factors, but because it arises from qualitative data rather than theoretical constructs, it provides detail and adds depth to the analysis of a PCP's decision-making process. It is also addresses the issue of visit type, and is current to the model of care delivery as practiced today.

\section{Limitations}

The interviews we conducted were in an academic setting and providers may be different than those in other practice environments. However, even within this system, providers practiced in a variety of clinical settings, and we believe our efforts to ensure internal reliability made this study exportable to other clinic-based settings. Interviews are an effective way of identifying and exploring perceptions that cannot be discovered with surveys or observation, but can nonetheless be biased. Although we attempted to validate our semi-structured interview guide and scenarios by pilot-testing before implementation, salient topics may have been omitted. Each interviewer was trained to arrange and word each interview question in the same manner but some flexibility was allowed in order to achieve depth of responses, thus potentially resulting in a variation of responses and weakening comparability of answers. In the development of major themes, it is always possible that some meanings could have been lost. Prior studies have shown that providers tend to over-estimate their delivery of preventive services (Woo et al., 1985; Montano and Phillips, 1995), but as our purpose was to seek decision-making insights of providers, we did not address actual rates of preventive health delivery. As this was a study of providers and their decision-making, it was 
necessarily provider-centric and although PCPs did report occasions in which the patient initiated preventive care requests, in this research we did not seek to address the patient voice. Our conceptual model arose from the thematic analysis, but it has not been tested in other clinical settings.

\section{Implications for practice}

As healthcare systems work toward improving delivery rates of preventive care, they may consider the insights this model provides regarding how PCPs make their decisions. Efforts to increase whether preventive care is delivered in a given clinic visit may be viewed from this lens: PCPs act pragmatically and attempt to triage appropriately, with multiple factors tending to lead to deferral of preventive services. More research is needed to determine whether addressing the barriers and facilitators for these factors may then improve preventive service delivery. This conceptual model of Pragmatic Deferral could be used to guide further research in improving clinic-based rates of preventive health delivery if behavior change on the part of providers is required. Efforts to steer what preventive services are delivered may also be challenging. As we found that there was no single guiding rubric for how a PCP prioritizes which service to address, external efforts to increase certain services will likely need to include the input of PCPs. Finally, as the team-based model grows in popularity, care systems may wish to consider the shared decision-making role that PCPs wish to retain.

In Essential of the U.S. Health Care System, 4th edition, authors Shi and Singh (2017) pointed out: 'The ideal role for primary care would include integrated healthcare in the form of comprehensive, coordinated and continuous services offered with a seamless delivery'. Our findings support and give additional substance to the PCP's role as principal in healthcare. Future qualitative research is needed to continue to understand how new healthcare systems affect decision-making of PCPs.

\section{Acknowledgments}

The authors acknowledge Ben Thompson and Nieves A. Martinez for assistance with transcription.

\section{Financial Support}

This research received no specific grant from any funding agency in the public, commercial, or notfor-profit sectors. C.J.W. was partially supported internally from the Division of General Internal Medicine by the Stern Family Fund.

\section{Conflicts of Interest}

None.

\section{Ethical Standards}

This research was approved by the University of Washington Institutional Review Board HSD no. 49491.

\section{References}

Abraham, T.H., Lewis, E.T., Drummond, K.L., Timko, C. and Cucciare, M.A. 2017: Providers' perceptions of barriers and facilitators to disclosure of alcohol use by women veterans. Primary Health Care Research \& Development 18, 64-72.

Boulware, L.E., Marinopoulos, S., Phillips, K.A., Hwang, C.W., Maynor, K., Merenstein, D., Wilson, R.F., Barnes, G.J., Bass, E.B., Powe, N.R. and Daumit, G.L. 2007: Systematic review: the value of the periodic health evaluation. Annals of Internal Medicine 146, 289-300.

Burack, R.C. 1989: Barriers to clinical preventive medicine. Primary Care 16, 245-50.

Chaloupka, F.J, Yurekli, A and Fong, G.T. 2012: Tobacco taxes as a tobacco control strategy. Tobacco Control 21, 172-80.

Cohen, D., DiCicco-Bloom, B, Strickland, P.O., Headley, A., Orzano, J., Levine, J., Scott, J. and Crabtree, B. 2004: Opportunistic approaches for delivering preventive care in illness visits. Preventive Medicine 38, 565-73.

Corbin, J. and Strauss, A. 2015: Practical considerations for getting started. In Knight, V., editor, Basics of qualitative research: Techniques and procedures for developing grounded theory. Thousand Oaks, CA: SAGE Publications, 31-56.

Cornuz, J., Ghali, W.A., Di Carlantonio, D., Pecoud, A. and Paccaud, F. 2000: Physicians' attitudes towards prevention: importance of intervention-specific barriers and physicians' health habits. Family Practice 17, 535-40.

Cosgrove, J.C. 2012: Medicare: use of preventive services could be better aligned with clinical recommendations. United States Government Accountability Office Report to Congressional Requesters. GAO-12-81 Medicare Preventive Services. Washington, D.C., USA.

Creswell, J.W. 2007: Qualitative inquiry and research design: Choosing among five approaches, second edition. Thousand Oaks, CA: SAGE Publications. 
Earp, J.A. and Ennett, S.T. 1991: Conceptual models for health education research and practice. Health Education Research 6, 163-71.

Edwards, T.C., Patrick, D.L., Topolski, T.D., Aspilnall, C.L., Mouradian, W.E. and Speltz, M.L. 2005: Approaches to craniofacial-specific quality of life assessment in adolescents. The Cleft Palate Craniofacial Journal 42, 19-24.

Elder, R.W. 2014: Combination of mass media health campaigns and health-related product distribution is recommended to improve healthy behaviors. American Journal of Preventive Medicine 47, 372-74.

Flocke, S.A., Stange, K.C. and Zyaznaski, S.J. 1998: The association of attributes of primary care with the delivery of clinical preventive services. Medical Care 36 (Suppl), AS21-S30.

Guerra, C.E., Jacobs, S.E., Holmes, J.H. and Shea, J.A. 2007: Are physicians discussing prostate cancer screening with their patients and why or why not? A pilot study. Journal of General Internal Medicine 22, 901-7.

Gupta, R., Dubé, K. and Bodenheimer, T. 2016: The road to excellence for primary care resident teaching clinics. Academic Medicine 91, 458-61.

Hing, E. and Albert, M. 2016: State variation in preventive care visits, by patient characteristics, 2012. NCHS Data Brief No. 234. Hyattsville, MD: National Center for Health Statistics.

Jaén, C.R., Stange, K.C. and Nutting, P.A. 1994: Competing demands of primary care: a model for the delivery of clinical preventive services. Journal of Family Practice 38, 166-71.

Kottke, T.E., Brekke, M.L. and Solberg, L.I. 1993: Making "time" for preventive services. Mayo Clinic Proceedings 68, 785-91.

Krogsboll, L.T., Jorgensen, K.J., Gronhoj Larsen, C. and Gotzsche, P.C. 2012: General health checks in adults for reducing morbidity and mortality from disease. Cochrane Database of Systematic Reviews, Issue 10. https://doi.org/ 10.1002/14651858.CD009009.pub2.

Medder, J.D, Kahn, N.B. Jr. and Susman, J.L. 1992: Risk factors and recommendations for 230 adult primary care patients, based on US Preventive Services Task Force guidelines. American Journal of Preventive Medicine 8, $150-153$.

Mirand, A.L., Beehler, G.P., Kuo, C.L. and Mahoney, M.C. 2003: Explaining the de-prioritization of primary prevention: physicians' perceptions of their role in the delivery of primary care. BMC Public Health 3, 15.

Montano, D.E. and Phillips, W.R. 1995: Cancer screening by primary care physicians: a comparison of rates obtained from physician self-report, patient survey, and chart audit. American Journal of Public Health 85, 795-800.

Mozaffarian, D., Benjamin, E.J., Go, A.S., Arnett, D.K., Blaha, M.J., Cushman, M., Das, S.R., de Ferranti, S., Després, J.-P., Fullerton, H.J., Howard, V.J., Huffman, M.D., Isasi, C.R., Jiménez, M.C., Judd, S.E., Kissela, B.M., Lichtman, J.H., Lisabeth, L.D., Liu, S., Mackey, R.H., Magid, D.J., McGuire, D.K., Mohler, E.R. III, Moy, C.S., Muntner, P., Mussolino, M.E., Nasir, K., Neumar, R.W., Nichol, G., Palaniappan, L.,
Pandey, D.K., Reeves, M.J., Rodriguez, C.J., Rosamond, W., Sorlie, P.D., Stein, J., Towfighi, A., Turan, T.N., Virani, S.S., Woo, D., Yeh, R.W. and Turner, M.B.; on behalf of the American Heart Association Statistics Committee and Stroke Statistics Subcommittee. 2016: Heart disease and stroke statistics-2016 update: a report from the American Heart Association. Circulation 133, e38-360.

Ockene, J.K., Edgerton, E.A., Teutsch, S.M., Marion, L.N., Miller, T., Genevro, J.L., Loveland-Cherry, C.J., Fielding, J.E. and Briss, P.A. 2007: Integrating evidencebased clinical and community strategies to improve health. American Journal of Preventive Medicine 32, 244-52.

Pelak, M., Pettit, A.R., Terwiesch, C., Gutierrez, J.C. and Marcus, S.C. 2015: Rethinking primary care visits: how much can be eliminated, delegated or performed outside of the face-to-face visit? Journal of Evaluation in Clinical Practice 21, 591-96.

Rebelsky, M.S., Sox, C.H., Dietrich, A.J., Schwab, B.R., Labaree, C.E. and Brown-McKinney, N. 1996: Physician preventive care philosophy and the five year durability of a preventive services office system. Social Science \& Medicine 43, 1073-81.

Rodriguez, H.P., Giannitrapani, K.F., Stockdale, S., Hamilton, A.B., Yano, E.M. and Rubenstein, L.V. 2014: Teamlet structure and early experiences of medical home implementation for veterans. Journal of General Internal Medicine 29 (Suppl 2), S623-631.

Rose, G.L., Ferraro, T.A., Skelly, J.M., Badger, G.J., MacLean, C.D., Fazzino, T.L. and Helzer, J.E. 2015: Feasibility of automatic pre-screening for lifestyle and behavioral health risk factors in primary care. BMC Family Practice 16, 150.

Roush, S.W. and Murphy, T.V., Vaccine-Preventable Disease Table Working Group. 2007: Historical comparisons of morbidity and mortality for vaccine-preventable diseases in the United States. JAMA 298, 2155-163.

Ruffin, M.T., Gorenflo, D.W. and Woodman, B. 2000: Predictors of screening for breast, cervical, colorectal, and prostatic cancer among community-based primary care practices. Journal of the American Board of Family Practice 13, 1-10.

Shi, L. and Singh, D.A. 2017: Essentials of the U.S. health care system, Fourth edition. Burlington, MA: Jones and Bartlett Learning.

Simmons, M., Guerra-Reyes, L., Meyerson, B., Adams, K. and Sanders, S. 2016: Exploring provider perspectives as barriers and facilitators to implementation of quality family planning recommendations at Title $X$ Clinics: a qualitative study. Womens Health Issues 26, 628-33.

Snipelisky, D., Carter, K., Sundsted, K. and Burton, M.C. 2016: Primary care physicians practicing preventive medicine in the outpatient setting. International Journal of Preventive Medicine 7, 5.

Society of General Internal Medicine. 2013: Choosing wisely. Retrieved 4 August 2016 fromhttp://www.choosingwisely. org/societies/society-of-general-internal-medicine/. Released September 12, 2013. 
Squires, H., Chilcott, J., Akehurst, R., Burr, J. and Kelly, M.P. 2016: A framework for developing the structure of public health economic models. Value Health 19, 588-601.

Stange, K.C., Flocke, S.A., Goodwin, M.A., Kelly, R.B. and Zyzanski, S.J. 2000: Direct observation of rates of preventive service delivery in community family practice. Preventive Medicine 31, 161-76.

Stange, K.C., Zyzanski, S.J., Smith, T.F., Kelly, R., Langa, D.M., Flocke, S.A. and Jaen, C,R. 1998: How valid are medical records and patient questionnaires for physician profiling and health services research? A comparison with direct observation of patient visits. Medical Care 36, 851-67.

Starks, H. and Trinidad, S.B. 2007: Choose your method: a comparison of phenomenology, discourse analysis, and grounded theory. Qualitative Health Research 17, 1372-380.

U. S. Department of Health and Human Services. 2016: Agency of healthcare research and quality. Defining the patient centered medical home. Retrieved 30 June 2016 from https://pcmh.ahrq.gov/page/defining-pcmh.
U. S. Preventive Services Task Force. 2017: USPSTF A and B recommendations. Retrieved 11 June 2017 from http://www. uspreventiveservicestaskforce.org/Page/Name/uspstf-a-and-brecommendations/.

Wong, C.J., Gaster, B. and Dugdale, D.C. 2014: Choosing wisely: in defense of the preventive health visit. American Journal of Preventive Medicine 47, 653-55.

Woo, B., Woo, B., Cook, E.F., Weisberg, M. and Goldman, L. 1985: Screening procedures in the asymptomatic adult: a comparison of physicians' recommendations, patients' desires, published guidelines, and actual practice. JAMA 254, 1480-484.

Yarnall, K.S., Pollak, K.I., Ostbye, T., Krause, K.M. and Michener, J.L. 2003: Primary care: is there enough for prevention? Journal of Public Health 93, 635-41.

Yoon, P.W., Bastian, B., Anderson, R.N., Collins, J.L. and Jaffe, H.W. 2014: Centers for Disease Control and Prevention (CDC). Potentially preventable deaths from the five leading causes of death-United States, 2008-2010. MMWR Morbidity and Mortality Weekly Report 63, 369-74. 